\title{
Are diet and feeding behaviours associated with the onset of and recovery from slow weight gain in early infancy?
}

\author{
Linda I. Hollén ${ }^{1 *}$, Zia ud Din ${ }^{1,2}$, Louise R. Jones ${ }^{1}$, Alan M. Emond ${ }^{1}$ and Pauline Emmett ${ }^{1}$ \\ ${ }^{1}$ Centre for Child and Adolescent Health, School of Social and Community Medicine, University of Bristol, \\ Oakfield House, Oakfield Grove, Bristol BS8 2BN, UK \\ ${ }^{2}$ Department of Human Nutrition, The University of Agriculture, Peshawar, Pakistan
}

(Submitted 13 June 2013 - Final revision received 14 November 2013 - Accepted 26 November 2013 - First published online 6 February 2014)

\section{Abstract}

Infants with slow weight gain cause concern in parents and professionals, but it is difficult to be certain whether such infants are genetically small or whether their energy intake is insufficient. The aim of the present study was to assess the impact of diet and feeding behaviours on slow weight gain early in infancy. The sample was 11499 term infants from the Avon Longitudinal Study of Parents and Children (ALSPAC). A total of 507 cases of slow weight gain from birth to 8 weeks were identified and the remaining 10992 infants were used as controls. It was found that infants who gained weight slowly between birth and 8 weeks were more likely to exhibit feeding problems such as weak sucking and slow feeding during this period. Feeding problems were substantially reduced during the recovery phase ( 8 weeks to 2 years) when these infants exhibited enhanced catch-up in weight. The proportion of mothers breast-feeding in the 4th week after birth was higher for slow weight gainers, but they were more likely to switch to formula at the start of recovery. During recovery, slow-weight gain infants had a slightly higher energy intake from formula and solids than controls. In conclusion, feeding problems seem to be the most important factors associated with the onset of early slow weight gain. Subsequently, a reduction of feeding problems and an increase in overall energy intake may contribute to their weight recovery. Health professionals should look for feeding problems in the first few weeks after birth and help mothers establish adequate feeding practices.

Key words: Infant nutrition: Weight faltering: Feeding behaviours: Avon Longitudinal Study of Parents and Children

Weight faltering, a relatively common growth pattern in infancy, is a term used to describe slow weight gain in children ${ }^{(1,2)}$. Previous research has shown that familial factors, such as parental height and parity ${ }^{(3)}$, and postnatal factors, such as infant sucking and health ${ }^{(4)}$, as well as problematic motherchild relationships ${ }^{(5)}$, are strongly associated with early weight faltering. Poor infant weight gain, at least in the UK, is, however, not associated with traditional markers of socio-economic deprivation $^{(3,4)}$. Early weight faltering is associated with outcomes such as developmental delay ${ }^{(6-8)}$, intelligence quotient deficits $^{(9,10)}$ and insecure attachment ${ }^{(11)}$. Therefore, identification of factors that could aid in the prevention of weight faltering in infancy is of crucial importance.

It is well known that dietary intake and nutrition throughout childhood are key determinants of growth and development and have a strong relationship with body size and composition. A Canadian cohort study ${ }^{(12)}$, for example, has reported accelerating effects of formula and other milks ( $v$. breast milk) on weight and length gain throughout infancy and displacement of milk feeding by cereals early on to adversely affect growth (also see Durmus et al. ${ }^{(13,14)}$ and van Rossem et al. $\left.{ }^{(14)}\right)$. Many recent studies have focused on diet- and feeding-related risk factors for rapid weight gain in infancy and subsequent childhood obesity, in particular, the potential protective effect of breast-feeding ${ }^{(15,16)}$, whereas far fewer studies have focused on detailed diet intake and feeding practices associated with slow weight gain ${ }^{(4,17)}$. The identification of feeding-related predictors that might increase the risk of slow weight gain in infancy may inform health professionals about potential preventive strategies to help mothers adopt appropriate infant feeding practices.

In the present study, we used data from the Avon Longitudinal Study of Parents and Children (ALSPAC) ${ }^{(18,19)}$, one of the few ongoing studies carried out in the developed world with detailed measurements of weight and dietary intake from infancy to childhood and into adolescence and adequate measures of confounding variables. Infants with early weight faltering have already been identified in the $\operatorname{ALSPAC}^{(3,4,20)}$. ALSPAC infants who gained weight slowly between birth and 8 weeks (period of weight faltering) exhibited an

Abbreviations: ALSPAC, Avon Longitudinal Study of Parents and Children; ESWG, early slow-weight gain; IQR, interquartile range. 
increased weight velocity compared with controls between 8 weeks and 2 years (recovery phase), resulting in their weight returning to the reference mean (British 1990 growth reference ${ }^{(21)}$ ) for that age ${ }^{(20)}$. After 2 years, this group gained weight at a rate similar to that of the control group, but remained slightly lighter and shorter than the control group at the age of 13 years (Fig. 1). Only 5.7\% of the infants exhibiting early weight faltering continued to gain weight slowly after 8 weeks of age, whereas the rest recovered. In the present study, we investigated whether diet and/or feeding behaviours differ between the two groups gaining weight at different rates in the first 2 months and between 2 months and 2 years. As a child's ability or eagerness to feed may be affected by the environment in which he or she lives (e.g. parenting ability and physical environment) and/or his or her personal/temperamental traits, we also investigated whether these variables differ between the two weight gain groups.

\section{Subjects and methods}

\section{Subjects}

The ALSPAC is a longitudinal prospective cohort study that recruited pregnant mothers in the former Avon Health Authority area with an expected date of delivery between 1 April 1991 and 31 December 1992, resulting in a cohort of 14062 live births. Details of the recruitment and representativeness of the ALSPAC cohort have been described elsewhere ${ }^{(19,21)}$. ALSPAC website $^{(19)}$ contains details of all available data through a fully searchable data dictionary (http://www.bristol.ac.uk/alspac/ researchers/data-access/data-dictionary/). Ethical approval was obtained from the ALSPAC Law and Ethics Committee and the Local Research Ethics Committees.

Details regarding the identification of infants with early weight faltering in the ALSPAC cohort have also been described elsewhere ${ }^{(3,4,10)}$. Briefly, weights recorded at birth and 8 weeks (range 1-3 months) by health professionals as part of the routine child health surveillance were extracted from the Avon
Child Health Computer System and standardised to $z$-scores (standard deviation score) adjusting for differences in sex and age (gestational age in weeks for weight at birth and infant age in weeks for weight at 8 weeks) using the British 1990 growth reference ${ }^{(22)}$. Weight gain was assessed by calculating the difference in $z$-scores between birth and 8 weeks, adjusting for regression towards the mean using correlates from within the cohort and Cole's equation ${ }^{(23)}$. Such conditional weight gain provides a more accurate measure of infant growth, as it accounts for the smaller infants who tend to grow faster and the larger infants who tend to grow slower ${ }^{(24)}$. It is also considered to be a more sensitive criterion for identifying children with weight faltering ${ }^{(25)}$. Infants with early weight faltering were defined as those below the 5 th centile for weight gain (standard deviation score $<-1.645$ ) between the two time points, and hereafter they are referred to as the early slow-weight gain (ESWG) group.

\section{Data}

The various time points at which variables used in the present study were obtained are summarised in Table 1.

\section{Diet and feeding behaviours}

Parent-reported questionnaires. After birth, parents were asked to fill in detailed questionnaires regarding their child's health and behaviour including a section on the child's eating habits (designed by an experienced dietitian; see Emmett $^{(26)}$ for details). Parents were asked about feeding methods (breast-fed or bottle-fed), age at the introduction of milk/solids, consumption frequency, and feeding behaviours. In the present study, we used questionnaires at ages 4 weeks, 6 months and 15 months. The questionnaire used at 4 weeks mainly included information on feeding methods and feeding behaviours, including difficulties. The questionnaire used at 6 months included questions about feeding behaviours referring to two time periods: those occurring between 0 and

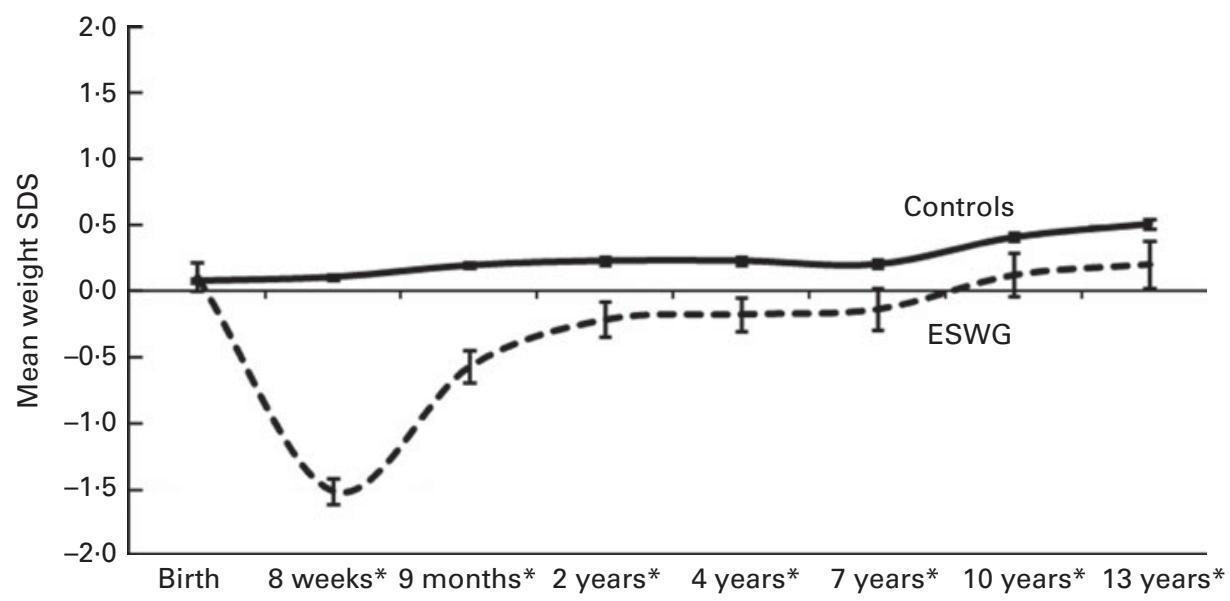

Fig. 1. Cross-sectional mean weight standard deviation scores (SDS) for early slow-weight gain (ESWG) and normal-weight gain (control) children from birth to 13 years in comparison with the UK 1990 reference $($ SDS $=0)$. ${ }^{*}$ Means of groups are different from each other $(P<0.01)$ at these ages $(n 11499-5115$; $100-44 \%)$. This graph was adapted from a version first published in Din et al. ${ }^{(20)}$. 
Table 1. Overview of Avon Longitudinal Study of Parents and Children (ALSPAC) data collection for variables used in the present study

\begin{tabular}{|c|c|c|c|}
\hline Age & Sample & Diet/feeding & Other \\
\hline 32 weeks of gestation & ALSPAC (mothers) & & $\begin{array}{l}\text { Parity } \\
\text { Maternal age } \\
\text { Maternal height } \\
\text { Maternal education } \\
\text { Ethnicity } \\
\text { Car use }\end{array}$ \\
\hline 4 weeks & ALSPAC & $\begin{array}{l}\text { Feeding methods } \\
\text { Feeding behaviour }\end{array}$ & Sex \\
\hline 4 months & Subsample CIF & $1 \mathrm{~d}$ diet record & \\
\hline 6 months & ALSPAC & $\begin{array}{l}\text { Infant FFQ } \\
\text { Feeding behaviour }\end{array}$ & $\begin{array}{l}\text { Home score } \\
\text { Parenting score } \\
\text { Temperament } \\
\text { Infant health }\end{array}$ \\
\hline $\begin{array}{l}8 \text { months } \\
15 \text { months }\end{array}$ & $\begin{array}{l}\text { Subsample CIF } \\
\text { ALSPAC }\end{array}$ & $\begin{array}{l}3 \mathrm{~d} \text { diet record } \\
\text { Infant FFQ }\end{array}$ & \\
\hline 18 months & $\begin{array}{l}\text { ALSPAC } \\
\text { Subsample CIF }\end{array}$ & $3 \mathrm{~d}$ diet record & Weight worry \\
\hline
\end{tabular}

3 months and those occurring between 4 and 6 months. The 0 -3-month data fall within the weight-faltering period, and they were, therefore, used to compare findings from the 4-week questionnaire. Questions asked at 15 months regarding feeding behaviours reflected problems occurring between 6 and 15 months. We also included a question about a mother's worry about her child's weight development extracted from a questionnaire at 18 months.

Diet diaries. Detailed dietary intake was assessed using diet diaries for a $10 \%$ subsample of the children (known as Children in Focus) at 4, 8 and 18 months. The carer completed the diaries by recording everything the child ate and drank in household measures for three $24 \mathrm{~h}$ periods (only one at 4 months). These data were used to calculate mean daily energy and nutrient intakes and amounts of various groups of foods consumed (details provided in Noble \& Emmett ${ }^{(27,28)}$ and Cowin \& Emmett $\left.{ }^{(29)}\right)$. To assess differences in dietary intake, we compared average intakes of protein, fat, carbohydrates, intrinsic/milk sugars and non-milk extrinsic sugars (intrinsic and extrinsic sugars at only 8 and 18 months) of the weight gain groups. The intake of non-milk extrinsic (added) sugars was calculated from that of total sugars by deducting that of all the sugars from fresh fruit, vegetables and milk and of part of those from tinned fruit, baked beans, tinned pasta, tomato-based sauces, yogurt and fromage frais. Intrinsic/milk sugars were the remainder of total sugars. Energy intakes of ninety different foods and beverages obtained from the dietary records were averaged, and these food sources were categorised into four main groups: solids (sum of all solid food groups; all ages); formula milk (at 4 and 8 months); other milks (cow, soya and goat; at 8 and 18 months); other non-milk drinks (fruit juices, water, soft drinks and ready-made baby drinks; at 18 months). The number of babies fed only breast milk in the ESWG group was too low to analyse them separately at any of the ages ( $n 7$ at 4 months). We compared mean intakes of energy from these different sources in the two weight gain groups for consumers only.

\section{Home environment and temperamental traits}

To measure the mother's and partner's parenting style, questions such as the frequency with which they played with their child and participated in a variety of activities such as singing, showing books and playing with toys were asked in the parent-reported questionnaire at 6 months. Questions regarding home environment, for example, language stimulation, physical environment and punishment, were adapted from the validated HOME inventory (Home Observation for Measurement of the Environment $\left.{ }^{(30)}\right)$. We used a combined 'parents and home' score (mother's parenting score, partner's parenting score and home score summed). The child's temperament was measured using the Carey Infant Temperament Scale ${ }^{(31)}$, which generates several subscales, each measuring a temperamental trait. We summed scores on mood, intensity, activity, rhythmicity, approach, adaptability, persistence, distractibility and threshold subscales to generate a continuous measure of 'temperament ${ }^{\text {(32), }}$, where higher scores reflect more difficult temperament.

\section{Statistical analyses}

To investigate whether feeding behaviours, duration of breastfeeding ( $0-3$ weeks and $>3$ weeks) and proportion of infants given supplementary water (all extracted from the 4-week questionnaire; Table 2) differed between the two weight gain groups, we used a categorical principal component analysis using CATPCA in SPSS for Windows version 18 (SPSS, Inc.). Principal component analysis as a statistical method is used to reduce the original set of variables to a smaller set of uncorrelated components that represent most of the variance found in the original data ${ }^{(33)}$. In categorical principal component analysis, an optimal scaling approach is used to allow variables to be scaled at different levels; categorical variables are optimally quantified in the specified dimensionality (nominal or ordinal) and, as a result, non-linear relationships between variables are modelled. The number of components to be retained was chosen based on a scree plot and the 
Table 2. Variables for feeding behaviour and feeding methods at 4 weeks (from parent-reported questionnaires) that were included in the categorical principal component analysis and the resulting components on which they were loaded (\% variance explained)

\begin{tabular}{|c|c|c|c|}
\hline Variables included & Component $1(21 \%)$ & Component $2(13 \%)$ & Component $3(11 \%)$ \\
\hline Weak sucking (always ${ }^{*}$, sometimes or none) & $0.602 \dagger$ & -0.260 & -0.171 \\
\hline Exhaustion while feeding (always ${ }^{*}$, sometimes or none) & $0.594 \dagger$ & -0.016 & 0.036 \\
\hline Slow feeding (always*, sometimes or none) & $0.658 \dagger$ & -0.255 & -0.294 \\
\hline Taking small quantities (always*, sometimes or none) & $0.574 \dagger$ & -0.411 & 0.023 \\
\hline Refusing milk (always ${ }^{\star}$, sometimes or none) & $0.449 \dagger$ & -0.159 & -0.007 \\
\hline Unsatisfied (always ${ }^{\star}$, sometimes or none) & 0.342 & 0.215 & 0.251 \\
\hline General feeding difficulties (very often ${ }^{\star}$, quite often or none) & $0.575 \dagger$ & -0.258 & -0.015 \\
\hline Breast-feeding duration ( $\leq 3$ weeks ${ }^{*}$ or $>3$ weeks) & 0.377 & $0.623 \dagger$ & -0.358 \\
\hline Supplementary water feeding (yes* or no) & 0.268 & $0.604 \dagger$ & -0.178 \\
\hline Fed on regular schedule (always ${ }^{\star}$, tried to or fed on demand) & 0.218 & $0.530 \dagger$ & -0.402 \\
\hline Choking (always*, sometimes or none) & 0.318 & 0.011 & $0.638 \dagger$ \\
\hline Drinking too fast (always ${ }^{*}$, sometimes or none) & $0 \cdot 192$ & 0.416 & $0.639 \dagger$ \\
\hline Dribbling (always ${ }^{\star}$, sometimes or none) & 0.393 & 0.305 & 0.336 \\
\hline
\end{tabular}

* Indicates reference category within each variable.

† Variables with loadings above 0.4 for the component with which they exhibited the strongest association.

eigenvalues (all retained components have eigenvalues $>1$ ). Variables with loadings above \pm 0.4 were considered to have a strong association with that component (variables with loadings below 0.2 were excluded). For each child, individual component scores were created, which were then used in subsequent analyses (all further analyses were conducted using STATA version 12; StataCorp). All component scores were approximately normally distributed and had a mean of 0 and a standard deviation of 1 . The extracted principal component scores were then used in binomial logistic regressions with the dependent variable indicating whether the infant was in the slowest-growing $5 \%$ of the cohort (ESWG) or not (controls). OR, 95\% CI and $P$ values (two-sided) are reported. The Hosmer and Lemeshow test was conducted for determining goodness of fit, using a $5 \%$ significance level. Depending on data distribution, other univariate analyses on questionnaire data were conducted using the $\chi^{2}$ test, unpaired $t$ tests or Mann-Whitney $U$ tests ( $z$-statistic). Analyses were carried out at a significance level of $0 \cdot 01$.

As familial, socio-economic and other parental factors (also obtained by parent-reported questionnaires) could affect the choices of foods and drinks that are given to a child ${ }^{(34)}$ and have been shown to be associated with slow weight gain in ALSPAC infants ${ }^{(3,4)}$, we included the following confounders in our analyses on questionnaire data: parity (single or multiple); maternal height (1st quintile, middle quintiles or 5 th quintile); maternal age $(<24,24-32$ or $>32$ years); maternal education level (none, vocational, academic qualification at 16 years, academic qualification at 18 years or degree); ethnicity (White, Asian or other); car use (yes or no). As boys and girls grow at different rates and sex differences in dietary intake and food preferences have been widely reported in the literature ${ }^{(35)}$, sex was controlled for in all analyses. Where appropriate, we also included infant health up to 8 weeks (healthy, minor problems or generally unwell) as a potential confounder. Although we suffered a loss of about $20 \%$ of the data when controlling for confounders, there were no differences in the proportion of missing data between the ESWG and control groups.

For the subsample with diet diaries, all diet variables were standardised to a mean of 0 and a standard deviation of
1 before analysis. Mean macronutrient intakes were adjusted for the current body weight of the children and analysed for differences between weight gain groups with multivariate multiple linear regressions controlling for total energy intake and sex (the sample size for the ESWG group was too low to include other confounders). Depending on data distribution, differences in mean energy intake from solids, formula, other milks and other drinks (also adjusted for current body weight) in consumers only were analysed using unpaired $t$ tests or Mann-Whitney $U$ tests.

\section{Results}

\section{Ascertainment}

Of the 14062 live births, ninety-two (0.7\%) were lost to follow-up mainly because of family relocation. We also excluded 1292 infants who had missing weight data in the first year of infancy, eighty-nine children with major congenital disorders (e.g. cerebral palsy, Down's syndrome, cleft palate and congenital heart disease), 871 pre/post-term infants, 184 twins and thirtyfive infants with standard deviation score above +4 and below -4 for birth weight or weight at 8 weeks (assumed as data entry mistakes), leaving 11499 infants for analysis. Of these, 507 were classed as exhibiting slow weight gain in the first 8 weeks of life and the remaining 10992 infants were used as controls (all above the 5 th centile). There was no difference in birth weight (ESWG: mean 3481 (SE 22.8) g; control: mean 3470 (sE 4.5) g; $t(11387)=-0.53, P=0.60)$ or length at birth (crown-heel length) (ESWG: mean 50.6 (SE 0.16) cm; control: mean $50.5(\mathrm{sE} 0.03) \mathrm{cm} ; t(5681)=-0.59, P=0.56)$ between the ESWG and controls groups.

Diet diaries were collected from 853, 1131 and 1026 children at 4,8 and 18 months, respectively (83, 86 and $83 \%$, respectively, of those seen at that clinic). Of these, 812 normal-growing children (421 boys and 391 girls) and fortyone slow-growing children (twenty-six boys and fifteen girls) at 4 months, 1023 normal-growing children (552 boys and 471 girls) and forty-eight slow-growing children (twenty-eight boys and twenty girls) at 8 months, and 868 normal-growing children (468 boys and 400 girls) and forty 
slow-growing children (twenty-two boys and eighteen girls) at 18 months were included in the study. A total of twenty-six of the slow-growing children and 566 of the normal-growing children had diet diaries completed at all three ages.

\section{Period of weight faltering}

Principal component analysis of data obtained from the questionnaire used at 4 weeks yielded three components explaining $45 \%$ of the variance in the original data (Table 2). Most variables related to feeding problems were most strongly loaded on principal component 1; breast-feeding duration, supplementary water feeding and being fed on a regular schedule were most strongly loaded on component 2, whereas choking and drinking too fast were most strongly loaded on component 3 (Table 2). Scores for all components differed between ESWG infants and controls (Table 3). At 4 weeks, ESWG infants were more likely than controls to exhibit feeding behaviours positively loaded on component 1 (weak sucking, exhaustion while feeding, slow feeding, taking small quantities, refusing milk and general feeding difficulties), but less likely to exhibit those positively loaded on component 2 (shorter breast-feeding duration, water supplement and being fed on a regular schedule) and component 3 (choking and drinking too fast; Table 3). All effects remained after controlling for confounders and after stratifying by current feeding method (breast-fed or bottlefed at 4 weeks; Table 3). The results of univariate analysis of data obtained for the $0-3$-month questions asked in the 6-month questionnaire (within the weight-faltering period) confirmed our findings from the 4-week questionnaire; ESWG infants were more likely than controls to feed slowly, take small quantities and refuse milk (Table 4).

\section{Recovery phase}

How infants feed. The association between slow feeding, taking small quantities and refusing milk between 0 and 3 months and being in the ESWG group was not apparent at 4-6 months of age (Table 5). Also, a derived feeding difficulty score combining five measures of feeding problems between 6 and 15 months (not eating enough, choosy, overeating, refusing food and lack of routine) did not differ between the groups (ESWG: median 2 (interquartile range (IQR) 3), $n$ 398; control: median 2 (IQR 3), $n$ 9077; $z=-1 \cdot 18, P=0 \cdot 24$ ). Even so, the proportion of mothers who were worried about their child's weight development for the ESWG group was twice as high as that for the control group at 18 months (ESWG: 37/ $407=9 \%$; control: $402 / 9163=4 \% ; \chi^{2} 19 \cdot 7, P<0 \cdot 001$ ).

What infants eat. In the 4 th week after birth, mothers of the ESWG group were more likely than those of the control group to stop breast-feeding at the beginning of the recovery phase (ESWG: 49/162=30\%; control: $615 / 2697=23 \% ; \chi^{2}$ $21 \cdot 6, P=0 \cdot 001)$. Although $96 \%$ of all children in the ALSPAC had started consuming solids by 4 months of age, slowweight gain children started slightly later than controls. For every 1-month increase in the age at which solid foods started being consumed, infants were 1.21 (95\% CI 1.08 , $1.35)$ times more likely to be in the ESWG group $(P<0 \cdot 001)$. This association remained after adjusting for all potential confounders $\left(n_{\mathrm{ESWG}}=378 ; n_{\text {Control }}=8379 ; 1 \cdot 25(1 \cdot 11,1 \cdot 41)\right)$. We found no difference between the groups in the number of breast-feeds (ESWG: median 4 (IQR 2), $n$ 112; control: median 4 (IQR 2), $n$ 2659; $z=0.52, \quad P=0.60$ ) or solid meals (ESWG: median 3 (IQR 0), $n$ 422; control: median 3 (IQR 0), $n$ 9424; $z=-0.57, P=0.57$ ) a child had per day at 6 months.

Table 3. Adjusted associations between 4-week principal component scores (Table 2) and the probability of being in the early slow-weight gain (ESWG) group*

(Odds ratios and $95 \%$ confidence intervals)

\begin{tabular}{|c|c|c|c|c|c|c|c|}
\hline & \multicolumn{2}{|c|}{ Adjusted $1 \dagger$} & \multicolumn{2}{|c|}{ Adjusted $2 \ddagger$} & \multicolumn{2}{|c|}{ Adjusted $3 \S$} & \multirow[b]{2}{*}{$n$ (ESWG/Controls)\| } \\
\hline & OR & $95 \% \mathrm{Cl}$ & OR & $95 \% \mathrm{Cl}$ & OR & $95 \% \mathrm{Cl}$ & \\
\hline \multicolumn{8}{|l|}{ Principal component 1} \\
\hline All feeding methods & $1 \cdot 29$ & $1 \cdot 19,1.40$ & $1 \cdot 26$ & $1 \cdot 16,1 \cdot 38$ & 1.24 & $1 \cdot 13,1 \cdot 36$ & $370 / 8225$ \\
\hline Breastfed at 4 weeks & 1.44 & $1.23,1.68$ & 1.45 & $1.24,1.71$ & 1.38 & $1 \cdot 16,1.64$ & $174 / 3718$ \\
\hline Bottlefed at 4 weeks & 1.45 & $1.29,1.63$ & 1.42 & $1 \cdot 26,1 \cdot 61$ & 1.41 & $1 \cdot 23,1.61$ & $127 / 3415$ \\
\hline \multicolumn{8}{|l|}{ Principal component 2} \\
\hline All feeding methods & 0.66 & $0.60,0.73$ & 0.65 & $0.58,0.72$ & 0.63 & $0.57,0.71$ & $370 / 8225$ \\
\hline Breastfed at 4 weeks & 0.66 & $0.53,0.82$ & 0.68 & $0.54,0.85$ & 0.66 & $0.52,0.83$ & $174 / 3718$ \\
\hline Bottlefed at 4 weeks & 0.59 & $0.50,0.69$ & 0.58 & $0.49,0.68$ & 0.58 & $0.48,0.69$ & $127 / 3415$ \\
\hline \multicolumn{8}{|l|}{ Principal component 3} \\
\hline All feeding methods & 0.74 & $0.66,0.82$ & 0.75 & $0.67,0.84$ & 0.74 & $0.66,0.83$ & $370 / 8225$ \\
\hline Breastfed at 4 weeks & 0.75 & $0.62,0.91$ & 0.76 & $0.63,0.92$ & 0.73 & $0.60,0.89$ & $174 / 3718$ \\
\hline Bottlefed at 4 weeks & 0.67 & $0.56,0.81$ & 0.66 & $0.55,0.80$ & 0.68 & $0.56,0.83$ & $127 / 3415$ \\
\hline
\end{tabular}

* The first analysis included infants who were breast-fed, bottle-fed or both (all feeding methods - not stratified). The second analysis was stratified by feeding method (breast-fed or bottle-fed at 4 weeks). Mixed feeders were included only in the group comprising all feeding methods as sample size was too low for ESWG infants.

$\dagger$ Model 1: adjusted for sex and infant health.

$\ddagger$ Model 2: model 1 + adjustment for maternal height and maternal age.

$\S$ Model 3: model $1+$ model 2 + adjustment for maternal education, car use, ethnicity and parity.

\| After full adjustment. 
Table 4. Associations between measures of feeding behaviour in the $0-3$ months after birth and the probability of being in the early slow-weight gain (ESWG) group*

(Odds ratios and $95 \%$ confidence intervals)

\begin{tabular}{|c|c|c|c|c|c|c|c|}
\hline \multirow[b]{2}{*}{ Variables } & \multicolumn{2}{|c|}{ Adjusted $1 \dagger$} & \multicolumn{2}{|c|}{ Adjusted 2‡ } & \multicolumn{2}{|c|}{ Adjusted $3 \S$} & \multirow[b]{2}{*}{$n$ (ESWG/Control)\| } \\
\hline & OR & $95 \% \mathrm{Cl}$ & OR & $95 \% \mathrm{Cl}$ & OR & $95 \% \mathrm{Cl}$ & \\
\hline Slow feeding & 1.78 & $1 \cdot 42,2 \cdot 23$ & 1.74 & $1 \cdot 38,2 \cdot 19$ & $1 \cdot 80$ & $1 \cdot 42,2 \cdot 27$ & $334 / 7521$ \\
\hline Taking small quantities & 1.39 & $1 \cdot 10,1 \cdot 76$ & 1.33 & $1.04,1.70$ & $1 \cdot 32$ & $1.03,1.69$ & $320 / 7045$ \\
\hline Unsatisfied & 0.99 & $0.79,1.23$ & 1.00 & $0.81,1.26$ & 0.99 & $0.79,1.25$ & $325 / 6974$ \\
\hline Choking & 0.52 & $0.32,0.85$ & 0.51 & $0.31,0.85$ & 0.46 & $0.27,0.79$ & $317 / 7114$ \\
\hline Refusing solids & 0.76 & $0.54,1.09$ & 0.77 & $0.53,1.10$ & 0.72 & $0.48,1.07$ & 312/6932 \\
\hline Refusing milk & 1.67 & $1 \cdot 22,2 \cdot 30$ & 1.62 & $1 \cdot 16,2 \cdot 25$ & 1.58 & $1 \cdot 12,2 \cdot 23$ & $313 / 6560$ \\
\hline Feeding routine & $1 \cdot 16$ & $0.84,1.61$ & $1 \cdot 15$ & $0.83,1.60$ & $1 \cdot 14$ & $0.81,1.60$ & $349 / 7669$ \\
\hline
\end{tabular}

* In the 6-month questionnaire, parents were asked about problems occurring during 0-3 months (weight-faltering period).

$\dagger$ Model 1: adjusted for sex and infant health.

$\ddagger$ Model 2: model 1 + adjustment for maternal height and maternal age.

$\S$ Model 3: model $1+$ model 2 + adjustment for maternal education, car use, ethnicity and parity.

\| After full adjustment.

In the subsample with diet diaries (for details, supplementary Table S1, available online), the average energy intake at 4 months was $2858(95 \%$ CI 2653, 3063) kJ/d (683 (95\% CI 634, $732) \mathrm{kcal} / \mathrm{d})$ and $2674(95 \% \mathrm{CI} 2632,2711) \mathrm{kJ} / \mathrm{d}(639(95 \% \mathrm{CI}$ $629,648) \mathrm{kcal} / \mathrm{d})$ for the ESWG $(n$ 40) and control $(n$ 812) groups, respectively. The respective values were 3594 (95\% CI $3381,3807) \mathrm{kJ} / \mathrm{d}(859(95 \% \mathrm{CI} 808,910) \mathrm{kcal} / \mathrm{d})$ and $3389(95 \%$ CI 3343,3435$) \mathrm{kJ} / \mathrm{d}(810(95 \%$ CI 799,821$) \mathrm{kcal} / \mathrm{d})$ at 8 months $\left(n_{\mathrm{ESWG}}=41 ; \quad n_{\text {Control }}=1023\right)$ and $4510 \quad(95 \%$ CI 4263 , $4761) \mathrm{kJ} / \mathrm{d}(1078(95 \% \mathrm{CI} 1019,1138) \mathrm{kcal} / \mathrm{d})$ and $4586(95 \%$ CI 4527,4648$) \mathrm{kJ} / \mathrm{d}(1096(95 \%$ CI 1082, 1111) kcal/d) at 18 months $\left(n_{\mathrm{ESWG}}=40 ; n_{\text {Control }}=868\right)$. Adjusted for body weight, the ESWG group had, on average, higher overall daily energy intake of $67 \mathrm{~kJ} / \mathrm{kg}$ ( $16 \mathrm{kcal} / \mathrm{kg}$ ) body weight at 4 months $(P<0.001)$ and $54 \mathrm{~kJ} / \mathrm{kg}(13 \mathrm{kcal} / \mathrm{kg})$ body weight $/ \mathrm{d}(P=0.006)$ higher energy intake from formula milk compared with the control group (consumers only: $n_{\mathrm{ESWG}}=34 ; n_{\text {Control }}=556$ ) . This difference persisted at 8 months for both overall energy intake $(54 \mathrm{~kJ} / \mathrm{kg}(13 \mathrm{kcal} / \mathrm{kg})$ body weight $/ \mathrm{d} ; P<0 \cdot 001)$ and formula milk (29 kJ/kg ( $7 \mathrm{kcal} / \mathrm{kg})$ body weight $/ \mathrm{d}$; only consumers: $\left.n_{\text {ESWG }}=40 ; n_{\text {Control }}=755 ; P=0.006\right)$. At 8 months, the ESWG group also had a $29 \mathrm{~kJ} / \mathrm{kg}(7 \mathrm{kcal} / \mathrm{kg})$ body weight $/ \mathrm{d}$ higher energy intake from solid foods compared with the control group $(P=0 \cdot 01)$. As a result, the group had a higher intake of macronutrients (protein, fat, carbohydrates and intrinsic/milk sugar) at 4 and 8 months ( $P<0.001$ for all).

Home environment, parenting and temperament. The combined 'parents and home' score did not differ between the ESWG and control groups (ESWG: median 10 (IQR 3), $n$ 426; control: median 10 (IQR 3), $n$ 9518; $z=0 \cdot 45$, $\left.n_{\mathrm{ESWG}}=426, \quad n_{\text {Control }}=9518, P=0.65\right)$. The 'temperament' score also did not differ between the two weight gain groups (ESWG: mean 179 (95\% CI 176, 182); control: mean $181(95 \%$ CI 180, 181); $t(9293)=1 \cdot 18, P=0 \cdot 24)$.

\section{Discussion}

In the present prospective population-based study, it was found that slow-weight gain infants were more likely than controls to exhibit feeding problems during the weightfaltering period immediately after birth. Weak sucking, slow feeding, taking small quantities, exhaustion while feeding, refusing milk and having more general feeding difficulties were more likely to be found in infants with slow weight gain regardless of being breast-fed, bottle-fed or both. All feeding problems were substantially reduced by 6 months of

Table 5. Associations between measures of feeding behaviour in the 4-6 months after birth and the probability of being in the early slow-weight gain (ESWG) group*

(Odds ratios and $95 \%$ confidence intervals)

\begin{tabular}{|c|c|c|c|c|c|c|c|}
\hline \multirow[b]{2}{*}{ Variables } & \multicolumn{2}{|c|}{ Adjusted 1† } & \multicolumn{2}{|c|}{ Adjusted $2 \ddagger$} & \multicolumn{2}{|c|}{ Adjusted $3 \S$} & \multirow[b]{2}{*}{$n$ (ESWG/Control) $\|$} \\
\hline & OR & $95 \% \mathrm{Cl}$ & OR & $95 \% \mathrm{Cl}$ & OR & $95 \% \mathrm{Cl}$ & \\
\hline Slow feeding & 0.80 & $0.50,1.29$ & 0.79 & $0.48,1.25$ & 0.76 & $0.46,1.26$ & $238 / 6456$ \\
\hline Taking small quantities & 0.83 & $0.58,1.19$ & 0.83 & $0.58,1.20$ & 0.80 & $0.55,1.16$ & $261 / 6374$ \\
\hline Unsatisfied & 0.83 & $0.60,1.16$ & 0.79 & $0.56,1.11$ & 0.81 & $0.57,1.15$ & $239 / 5266$ \\
\hline Choking & 0.97 & $0.71,1.33$ & 1.03 & $0.75,1.41$ & 1.00 & $0.73,1.39$ & $348 / 7436$ \\
\hline Refusing solids & 0.87 & $0.65,1.16$ & 0.86 & $0 \cdot 64,1 \cdot 16$ & 0.89 & $0.65,1.20$ & $347 / 7712$ \\
\hline Refusing milk & 0.75 & $0.40,1.43$ & 0.78 & $0.41,1.48$ & 0.80 & $0.42,1.52$ & $282 / 6275$ \\
\hline Feeding routine & 0.52 & $0 \cdot 23,1 \cdot 18$ & 0.46 & $0 \cdot 19,1 \cdot 11$ & 0.39 & $0.14,1.06$ & $313 / 7103$ \\
\hline
\end{tabular}

* In the 6-month questionnaire, parents were asked about problems occurring during 4-6 months (recovery phase).

† Model 1: adjusted for sex and infant health.

$\ddagger$ Model 2: model 1 + adjustment for maternal height and maternal age

$\S$ Model 3: model $1+$ model 2 + adjustment for maternal education, car use, ethnicity and parity.

\| After full adjustment. 
age, when differences between the weight gain groups were no longer apparent. Slow-weight gain infants were introduced slightly later to solids compared with control infants. During the recovery phase, the detailed diet diaries collected from the subsample at 4 and 8 months revealed a difference in overall energy intake and intake from formula milk and solids with slow-weight gain infants exhibiting slightly higher intakes of $29-67 \mathrm{~kJ} / \mathrm{kg}$ body weight per $\mathrm{d}$.

Feeding problems in infants seem to be relatively common in the UK. The UK Infant Feeding Survey $2010^{(36)}$ revealed that about three in ten mothers had experienced some kind of feeding problem in the early weeks after delivery, with the most commonly reported problems being that their baby was not feeding sufficiently well, the baby being ill or not having enough breast milk. An earlier ALSPAC analysis ${ }^{(4)}$ also found that weak sucking was the most important symptom for both breast-fed and bottle-fed slow-weight gain infants in the first 8 weeks of life $30 \%$ in slow-growing infants compared with $15 \%$ in controls). There are a number of reasons for feeding problems occurring. There is a known association between a mother's mental health (depression and anxiety) and feeding difficulties, where children of depressed mothers are more likely to exhibit problems with feeding ${ }^{(37-40)}$. However, another ALSPAC analysis has shown no difference in the prevalence of maternal depression in the weightfaltering group compared with controls $^{(41)}$. Relationship problems between mother and child could potentially also affect feeding and have been shown to be associated with the onset of weight faltering in the first 4 months of life in a Danish cohort ${ }^{(5)}$. However, in the present study, we found no difference in the score combining parenting ability and home environment between the slow-weight gain and control infants. Another possibility is that a child's temperament affects feeding ${ }^{(40,42)}$. It has been shown previously that ALSPAC infants with difficult temperament have more feeding difficulties at both 1 and 6 months ${ }^{(40)}$, but the present results indicated no difference in temperamental traits between the ESWG and control groups. Finally, feeding problems and associated slow weight gain in the early weeks could be a marker of neurological impairment or oromotor dysfunction ${ }^{(43)}$. A slightly lower intelligence quotient at the age of 8 weeks in ESWG children compared with controls has been found previously in the ALSPAC ${ }^{(10)}$. Therefore, data from the ALSPAC overall suggest that the feeding behaviours associated with ESWG in these infants are more likely to be a marker of biological vulnerability, especially in terms of neurological impairment, than to be driven by parental factors such as depression and parenting, home environment or infant's temperament.

While we do not have detailed dietary data during the weight-faltering period, we found some evidence that diet may be important in recovery from slow growth. Mothers of slow weight gainers were more likely to stop breast-feeding and change to formula milk at the start of recovery, and in the subsample with diet diaries, we found a difference in formula and solid food intakes between the weight gain groups relatively early in the recovery phase. The differences we found, i.e. $54 \mathrm{~kJ} / \mathrm{kg}$ ( $13 \mathrm{kcal} / \mathrm{kg}$ ) body weight $/ \mathrm{d}$ at 4 months and $29 \mathrm{~kJ} / \mathrm{kg}(7 \mathrm{kcal} / \mathrm{kg})$ body weight $/ \mathrm{d}$ at 8 months, equate to 331 and $243 \mathrm{~kJ}$ (79 and $58 \mathrm{kcal}$ ) higher intake per day for slow-weight gain infants (based on the average weight of a slow-weight gain infant at 4 and 8 months, respectively), representing $7-12 \%$ of these infants' total energy intake per day at that age. These differences in energy intake are reflected in the higher intakes of protein, fat, carbohydrates and intrinsic and milk sugars we found in slow weight gainers at 4 and 8 months. Although we cannot say for sure, it is possible that the higher overall energy intake was enough to contribute to the increased growth rate (Fig. 1) observed in the ESWG group during the recovery phase.

Although we do not present and discuss results regarding specific effects of confounders herein, they do conform to previous research ${ }^{(3,4,20)}$ showing that slow weight gain in the first few weeks of life is independently associated with maternal height (shorter mothers), maternal age (older mothers), use of a car (no use of a car in the household), infant health (less healthy) and sex (dietary intakes of food, energy and many nutrients higher in boys than in girls ${ }^{(44)}$ ), whereas other socio-economic markers such as maternal education and ethnicity have no independent effects on slow weight gain. Although the amount of missing data on certain confounders was quite large (between 6 and 20\%), there were no differences in the proportion of missing data between the ESWG and control groups.

We investigated the association between diet and feeding behaviours and ESWG in infancy using a large representative cohort and prospectively collected feeding data, thus avoiding the biases inherent in clinically referred samples of children with weight faltering. The findings from the questionnaire used when children were 4 weeks of age were confirmed by the separate questionnaire used 5 months later, thus increasing confidence in these data. There was inevitably loss to follow-up as the children grew up, although every effort was made by the ALSPAC team to minimise this. Furthermore, we had only limited information about dietary intake during the weight-faltering period and so cannot comment on differences in energy and nutrient intakes occurring in this period. Detailed measures of dietary intake started being collected only at 4 months of age when growth recovery had started and were available only for a subsample of ALSPAC children who had been invited to research clinics at this age. This means that the dietary assessment data were available only for a very small group of slow-weight gain infants, thus limiting the power of the analyses to detect differences. Although diet diaries are considered as one of the best methods for self-reported dietary assessment ${ }^{(45)}$, we had to rely on untrained parental report of the foods/drinks being consumed by children. For infants aged 4 months, dietary intake was recorded for only $1 \mathrm{~d}$. Although recording for further days may have been preferred, infants at this age exhibit little day-to-day variation in the types of food eaten $^{(46)}$. As a decision was made to not overburden parents, we emphasised that they keep a shorter but accurate food record. Other biases would have been introduced during staff interpretation of the written records, the estimation of portion sizes and amounts of breast milk consumed, and the 
use of standard food tables for nutrient intake analysis. Nevertheless, these biases were the same for both groups of infants compared in the present study. Currently, we do not have information about any intervention from health professionals that may have changed feeding practices in these slowweight gain infants; however, these data may be available in the future through linkage to health service records. It is worth noting that the findings of the present study may not be relevant to slow weight gain occurring later in the first year as investigated by many others. Although a group of infants with weight faltering between 8 weeks and 9 months have been identified in the ALSPAC, this group is the subject of another paper.

In conclusion, it seems that feeding problems after birth are the most likely predictors of ESWG. Once these problems are resolved, these infants gain weight at a slightly faster rate than controls up to the age of 2 years, potentially fulfilling their genetic potential ${ }^{(44)}$. Being smaller, and therefore maybe relatively slower in their oromotor development, may explain why slow-weight gain infants were introduced to solids slightly later than controls. At the time the present study was carried out, mothers were advised to commence feeding solid foods to their children at about 3-4 months of age ${ }^{(47)}$ and very few infants in the study were introduced to solid foods after this. In light of current recommendations to exclusively breast-feed and to delay the introduction of solid foods to about 6 months of age ${ }^{(48)}$, health professionals in contact with infants in the first few months after birth should be aware of various feeding problems and focus on helping mothers establish adequate feeding practices. Although the proportion of worried mothers was relatively low, mothers of slow-weight gain infants were more worried about their child's weight gain compared with those of controls. To avoid such worry causing mothers to give up on breast-feeding or causing any other issues, mothers will need individualised advice and support if feeding problems persist and some reassurance can be given that children usually catch up in growth over time.

\section{Supplementary material}

To view supplementary material for this article, please visit http://dx.doi.org/10.1017/S0007114513004182

\section{Acknowledgements}

The authors are extremely grateful to all the families who took part in the present study, the midwives for their help in recruiting them and the whole ALSPAC team, including interviewers, computer and laboratory technicians, clerical workers, research scientists, volunteers, managers, receptionists and nurses.

The UK Medical Research Council (grant ref: 74882), the Wellcome Trust (grant ref: 076467) and the University of Bristol provided core support for the ALSPAC. The present study was supported by a grant from Wyeth Nutrition, UK. Wyeth Nutrition, UK, had no role in the study design and analysis or in the writing of this article.
The authors' contributions are as follows: L. I. H. prepared data for analyses, carried out the statistical analyses and wrote the manuscript; Z. D. prepared data for analyses; P. E. formulated the research questions and edited the manuscript; L. R. J. edited the manuscript; A. M. E. designed the ALSPAC weight faltering study and edited the manuscript.

P. E. has received research and consultancy funding from Pfizer Nutrition, Wyeth Nutrition, Danone and Plum Baby. Other authors declare no conflicts of interest.

\section{References}

1. Skuse D (1993) Epidemiological and definitional issues in failure to thrive. Child Adolesc Psychiatr Clin 2, 37-59.

2. Wright CM, Waterston A, Matthews J, et al. (1994) What is the normal rate of weight gain in infancy? Acta Paediatr $\mathbf{8 3}$, 351-356.

3. Blair PS, Drewett RF, Emmett PM, et al. (2004) Family, socioeconomic and prenatal factors associated with failure to thrive in the Avon Longitudinal Study of Parents and Children (ALSPAC). Int J Epidemiol 33, 1-9.

4. Emond AM, Blair PS, Drewett RF, et al. (2007) Postnatal factors associated with failure to thrive in term infants in the Avon Longitudinal Study of Parents and Children. Arch Dis Child 92, 115-119.

5. Olsen EM, Skovgaard AM, Weile B, et al. (2010) Risk factors for weight faltering in infancy according to age of onset. Paediatr Perinat Epidemiol 24, 370-382.

6. Black M, Hutcheson J, Dubowitz H, et al. (1994) Parenting style and developmental status among children with nonorganic failure to thrive. J Pediatr Psychol 19, 689-707.

7. Skuse D, Pickles A, Wolke DF, et al. (1994) Postnatal growth and mental development: evidence for a sensitive period. $J$ Child Psychol Psychiatry 35, 521-545.

8. Wilensky D, Ginsberg G, Altman M, et al. (1996) A community based study of failure to thrive in Israel. Arch Dis Child 75, $145-148$.

9. Rudolf MCJ \& Logan S (2005) What is the long term outcome for children who fail to thrive? A systematic review. Arch Dis Child 90, 925-931.

10. Emond AM, Blair PS, Emmett PM, et al. (2007) Weight faltering in infancy and IQ levels at 8 years in the Avon Longitudinal Study of Parents and Children. Pediatrics 120, e1051.

11. Ward M, Lee S \& Lipper E (2000) Failure to thrive is associated with disorganized infant-mother attachment and unresolved maternal attachment. Inf Ment Health J 21, 428-442.

12. Kramer MS, Guo T, Platt RW, et al. (2004) Feeding effects on growth during infancy. J Pediatr 145, 600-605.

13. Durmuss B, van Rossem L, Duijts L, et al. (2011) Breastfeeding and growth in children until the age of 3 years: the Generation R Study. Br J Nutr 105, 1704-1711.

14. van Rossem LV, Kiefte-de Jong JC, Looman CW, et al. (2012) Weight change before and after the introduction of solids: results from a longitudinal birth cohort. Br J Nutr 109, $370-375$.

15. Armstrong J \& Reilly JJ (2002) Breastfeeding and lowering the risk of childhood obesity. Lancet 359, 2003-2004.

16. Schack-Nielsen L, Sorensen T, Mortensen EL, et al. (2010) Late introduction of complementary feeding, rather than duration of breastfeeding, may protect against adult overweight. Am J Clin Nutr 91, 619-627.

17. Wright CM, Parkinson KN \& Drewett RF (2005) How does maternal and child feeding behaviour relate to weight gain 
and failure to thrive? Data from a prospective birth cohort. Pediatrics 117, 1262-1269.

18. Golding J, Pembrey M, Jones R, et al. (2001) ALSPAC: the Avon Longitudinal Study of Parents and Children. I. Study methodology. Paediatr Perinat Epidemiol 15, 74-87.

19. Avon Longitudinal Study of Parents and Children (2013) Avon Longitudinal Study of Parents and Children. http:// www.bristol.ac.uk/alspac

20. Din uZ, Emmett P, Steer C, et al. (2013) Growth outcomes of weight faltering in infancy in ALSPAC. Pediatrics 131, e843-e849.

21. Boyd A, Golding J, Macleod J, et al. (2012) Cohort Profile: the 'children of the 90s' - the index offspring of the Avon Longitudinal Study of Parents and Children. Int J Epidemiol 42, 111-127.

22. Freeman JV, Cole TJ, Chinn S, et al. (1995) Cross sectional stature and weight reference curves for the UK, 1990. Arch Dis Child 73, 17-24.

23. Cole TJ (1995) Conditional reference charts to assess weight gain in British infants. Arch Dis Child 73, 8-16.

24. Heffer RW \& Kelly ML (1994) Nonorganic failure to thrive, developmental outcomes and psychosocial assessment and intervention issues. Res Dev Disabil 15, 247-268.

25. Edwards AGK, Halse PC, Parkin JM, et al. (1990) Recognising failure to thrive in early childhood. Arch Dis Child 65 $1263-1265$.

26. Emmett P (2009) Dietary assessment in the Avon Longitudinal Study of Parents and Children. Eur J Clin Nutr 63, Suppl. 1, S38-S44.

27. Noble S \& Emmett P (2001) Food and nutrient intake in a cohort of 8-month-old infants in the south-west of England in 1993. Eur J Clin Nutr 55, 698-707.

28. Noble S \& Emmett P (2006) Differences in weaning practice, food and nutrient intake between breast- and formula-fed 4-month-old infants in England. J Hum Nutr Diet 19, 303-313.

29. Cowin I \& Emmett P (2000) Diet in a group of 18-month children in South West England, and comparison with the results of a national survey. J Hum Nutr Diet 13, 87-100.

30. Bradley RH \& Caldwell BM (1981) The HOME inventory: a validation of the preschool scale for black children. Child Dev 52, 708-710.

31. Carey WB \& McDevitt SC (1977) Infant Temperament Questionnaire. Philadelphia, PA: Department Educational Psychology, Temple University.

32. Thomas A, Chess S \& Birch HG (1968) Temperament and Behaviour Disorder in Children. New York: University Press.

33. Kline P (1994) An Easy Guide to Factor Analysis. London: Routledge.

34. Smithers LG, Brazionis L, Golley RK, et al. (2012) Associations between dietary patterns at 6 and 15 months of age and socio-demographic factors. Eur J Clin Nutr 66, 658-666.
35. Wardle J, Haase AM \& Steptoe A (2004) Gender differences in food choice: the contribution of health beliefs and dieting. Ann Behav Med 27, 107-116.

36. The Information Centre, Government Statistical Service (2012) Infant Feeding Survey 2010 http://www.ic.nhs.uk/ pubs/infantfeeding10final

37. Stein A, Woolley H, Cooper SD, et al. (1994) An observational study of mothers with eating disorders and their infants. J Child Psychol Psychiatry 35, 733-748.

38. Stein A, Woolley H, Murray L, et al. (2001) Influence of psychiatric disorder on the controlling behaviour of mothers with 1-year-old infants. A study of women with maternal eating disorder, postnatal depression and a healthy comparison group. Br J Psychiatry 179, 157-162.

39. Wright CM, Parkinson KN \& Drewett RF (2006) The influence of maternal socioeconomic and emotional factors on infant weight gain and weight faltering (failure to thrive), data from a prospective birth cohort. Arch Dis Child 91, 312-317.

40. Micali N, Simonoff E, Stahl D, et al. (2011) Maternal eating disorders and infant feeding difficulties: maternal and child mediators in a longitudinal general population study. J Child Psychol Psychiatry 52, 800-807.

41. Drewett R, Blair P, Emmett P, et al. (2004) Failure to thrive in the term and preterm infants of mothers depressed in the postnatal period: a population-based birth cohort study. J Child Psychol Psychiatry 45, 359-366.

42. Lindberg L, Bohlin G \& Hagekull B (1994) Early food refusal: infant and family characteristics. Inf Ment Health $J \mathbf{1 5}$, 262-277.

43. Sullivan PB, Lambert B, Rose M, et al. (2000) Prevalence and severity of feeding and nutritional problems in children with neurological impairment: Oxford Feeding Study. Dev Med Child Neurol 42, 674-680.

44. Emmett P, Rogers I \& Symes C (2002) Food and nutrient intakes of a population sample of 3 -year-old children in the south west of England in 1996. Public Health Nutr 5 , 55-64.

45. Bingham SA, Cassidy A, Cole TJ, et al. (1995) Validation of weighed records and other methods of dietary assessment using the $24 \mathrm{~h}$ urine nitrogen technique and other biological markers. Br J Nutr 73, 531-550.

46. Paul AA, Black AE, Evans J, et al. (1988) Breastmilk intake and growth in infants from two to ten months. J Human Nutr 1, 437-450.

47. Department of Health (1988) Present day practice in infant feeding: third report. In Report on Health and Social Subjects No. 32. London: HMSO

48. Department of Health (2003) Infant feeding recommendation. http://www.dh.gov.uk/en/Publicationsandstatistics/Publicat ions/PublicationsPolicyAndGuidance/DH_4097197 